\title{
Anti-LAG-3 Monoclonal Antibody INCAGN02385
}

National Cancer Institute

\section{Source}

National Cancer Institute. Anti-LAG-3 Monoclonal Antibody INCAGN02385. NCI

Thesaurus. Code C157127.

A Fc-engineered immunog lobulin G1-kappa (IgG1k) monoclonal antibody targeting the co-inhibitory receptor lymphocyte-activation gene 3 protein (LAG-3; LAG3), with potential immune checkpoint inhibitory and antineoplastic activities. Upon intravenous administration, monoclonal antibody INCAGN02385 targets and binds to human LAG-3 on tumor-infiltrating lymphocytes (TILs) and blocks the interaction between LAG-3 and major histocompatibility complex class II (MHC II) molecules on the surface of antigenpresenting cells (APCs) and tumor cells. This prevents the negative regulation of T-cell activity that occurs via LAG-3-MHC II binding and enhances a cytotoxic T-lymphocyte (CTL)-mediated immune response against tumor cells, which leads to a reduction in tumor growth. LAG-3, a member of the immunog lobulin superfamily (IgSF), negatively regulates both proliferation and activation of T-cells. Its expression on TILs is associated with tumor-mediated immune suppression. 